\title{
DWI as a biomarker of renal function in children with CKD: what is the potential?
}

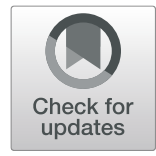

\author{
Sally Emad-Eldin ${ }^{*}$ D, Sunil Yadav ${ }^{1}$, Rasha Essam Eldin Galal ${ }^{2}$ and Wessam Abdelrahman Elzayat ${ }^{1}$
}

\begin{abstract}
Background: Evaluation of renal microstructure is pivotal for diagnosing and monitoring chronic renal disease. DWI has been proved to be practicable and reliable examination for the assessment of renal function and parenchymal damage in some renal diseases. Our aim is to appraise DWI sequence and ADC measurement as a potential tool of renal function assessment as well as establishing a possible relationship between the different CKD stages and the renal parenchymal ADC values changes.

Results: Regarding the cause of CKD, nine patients (45\%) had glomerulonephritis, 5 patients (25\%) had hemolytic uremic syndrome, 2 patients (10\%) had lupus nephritis, 2 patients (10\%) had nepheronophthisis, and 1 patient (1.5\%) had infantile nepherosis, whereas the cause of CKD was unknown in 1 patient (1.5\%).

The stages of CKD were classified according to KIDGO guidelines: 6 patients (30\%) were stage 1, 4 patients (20\%) were stage 2, 3 patients (15\%) were stage 3, 2 patients (10\%) were stage 4, and 5 patients (25\%) were stage 5 . The patients' group (group A) had a mean ADC value $\left(1.85 \times 10^{-3} \pm 0.24\right)$ which was significantly lower than that of the control group (group B) $\left(2.21 \times 10^{-3} \pm 0.12\right)$. As for the correlation between stage of CKD and ADC, we found it to be a moderate negative one with $r^{\prime}$ value of -.655 and a significant $p$ value of $<0.001$.

Conclusion: DWI is recognized as a promising imaging tool that can take part in the assessment of the morphological and functional changes in diffuse renal parenchymal disease, hence playing an important role in the early diagnosis and staging of chronic kidney disease.
\end{abstract}

Keywords: DWI, ADC, CKD, Children, Renal function

\section{Background}

Chronic kidney disease (CKD) is a significant health problem. Although relatively infrequent in the pediatric age group, it can be a devastating illness with numerous life-long consequences [1]. Serum markers such as blood urea nitrogen level, creatinine, and estimated glomerular filtration rate (eGFR) are useful parameters for estimating renal function [2]; however, the blood tests depend on age and body mass index; additionally, it cannot be used to evaluate a single kidney function [3].

Because of the limitations of serum markers, nowadays, imaging modalities have gained importance in the

\footnotetext{
* Correspondence: sallyemad@hotmail.com

'Diagnostic and Intervention Radiology Department, Cairo University

Hospitals, Kasr Al-Ainy, El-Manial, Cairo 11956, Egypt

Full list of author information is available at the end of the article
}

evaluation of renal function [4]. US and CT examinations can provide good anatomic images but provide insufficient functional information. Even though USG shows changes in renal echogenicity, which gives an impression of function, it has operator dependency and lacks objectivity; as for CT scan, it has exposure to ionizing radiation and may require use of iodinated contrast material, which is undesirable in patients with renal dysfunction $[4,5]$.

Diffusion-weighted imaging (DWI) is a technique used to display and measure the diffusion of water molecule. It reflects the Brownian motion of water molecules in the tissues [6]. Because of the kidney's task in water reabsorption and concentration dilution, water transport is considered the main renal function. The diffusion characteristics of the kidney may provide information on the 
mechanism of different renal pathology, like renal parenchymal disease, renal scarring, obstructive uropathy, and chronic renal failure [7].

Till date, there have been few studies investigating the use of DWI for functional assessment of the kidneys in the pediatric population [6-9].

Our aim is to appraise DWI sequence and ADC measurement as a potential tool of renal function assessment as well as establishing a possible relationship between the different CKD stages and the renal parenchymal $\mathrm{ADC}$ values changes.

\section{Methods}

\section{Study population}

This prospective study included 20 patients (12 males and 8 females) diagnosed with CKD. These patients were recruited from the outpatient CKD clinic and inpatients unit of pediatric nephrology and rheumatology department. Their ages ranged from 20 months to 18 years (mean age $10 \pm 5.80$ ). Our control group included 10 healthy children of matching age.

The study was approved by the Hospital Ethical Committee. Informed written consents were obtained from guardians of children. The clinical diagnosis was obtained from the treating nephrologists and clinical records of the patient.

Renal US and color Doppler were performed in all patients for initial disease evaluation and elucidate the diagnosis. Healthy control subjects were selected from the patients who presented to the Radiology department with various complains and siblings of cases of CKD who already had the result of their renal function test and urine analysis done for various reasons.

\section{Methods}

\section{Clinical examination}

The patients were examined clinically by the nephrologist in their respective clinics or units. The classification and stratification of the degree of CKD were according to KDIGO (Kidney Disease Improving Global Outcomes) guidelines $[10,11]$.

The patients were referred to the specialized CKD laboratory in pediatric nephrology department for renal function tests (serum creatinine, blood urea nitrogen, serum electrolytes, and in required cases of total protein, serum albumin, etc.) and urine analysis. The normal value according to the age and estimated GFR of each patient was analyzed by a nephrologist.

\section{Ultrasonography and Color Doppler examination}

Renal ultrasound and Doppler examination were performed for all patients, using an S-6 General Electric (USA) ultrasound device equipped with $5-12 \mathrm{MHz}$ high frequency transducer.
In all patients, renal size, site, echogenicity, corticomedullary differentiation, back pressure changes, and any other pathologically evident changes were considered.

Additionally, the resistivity index (RI) of segmental renal arteries was measured in upper, mid, and lower poles of both kidneys. All three values are taken, and it is calculated for each kidney, and at least two Doppler waveforms had to be obtained from a segmental renal artery.

\section{MRI imaging}

\section{A-imaging protocol}

All MRI studies were performed with $1.5 \mathrm{~T}$ two MR machines (Achieva and Intera; Philips Medical Systems, The Netherlands) using phased array abdominal coil and surface coils in smaller children.

All patients were examined in the supine position. Patients younger than 6 years old were sedated using oral chloral hydrate $(10 \%$ syrup, $100 \mathrm{mg} / \mathrm{ml})$ at a dose of 1 $\mathrm{mg} / \mathrm{kg}$.

Conventional MRI sequences including axial and coronal T2 FSE and axial T1 sequences were performed. This was followed by respiratory trigger echo-planar DW images in axial plane. Diffusion gradient $b$ values of 0 and $600 \mathrm{~s} / \mathrm{mm}^{2}$ were used.

\section{B-MRI data analysis}

The DWI data analysis and automatic generation of the ADC were carried on an independent workstation (Philips MR extended workspace, software version 2013). ADC map of the images was automatically generated.

Circular regions of interest (ROIs) measuring about $100 \mathrm{~mm}^{2}$ were used for quantitative analysis of the ADC values of the renal parenchyma. ROIs were placed at the renal parenchyma, not separately at the renal cortex and medulla.

We calculated three ADC measurements at the same level and used their mean values for data analysis.

\section{Statistical analysis}

The 25th version of the statistical package SPSS (Statistical Package for the Social Sciences) was used for data analysis. Mean, $+/-$ standard deviation, and median were used for quantitative data expression. Categorical data were expressed by frequency (count) and relative frequency (\%). We used non-parametric Mann-Whitney test to compare between quantitative variables [12], whereas chi-square $(x 2)$ was used for categorical data comparison. When the anticipated frequency was smaller than 5, exact test was applied [13]. Spearman correlation coefficient was used for correlative analysis between quantitative variables [14]. The $P$ values determined by chi-square test were regarded of statistical significance if ${ }^{<} 0.05$. 

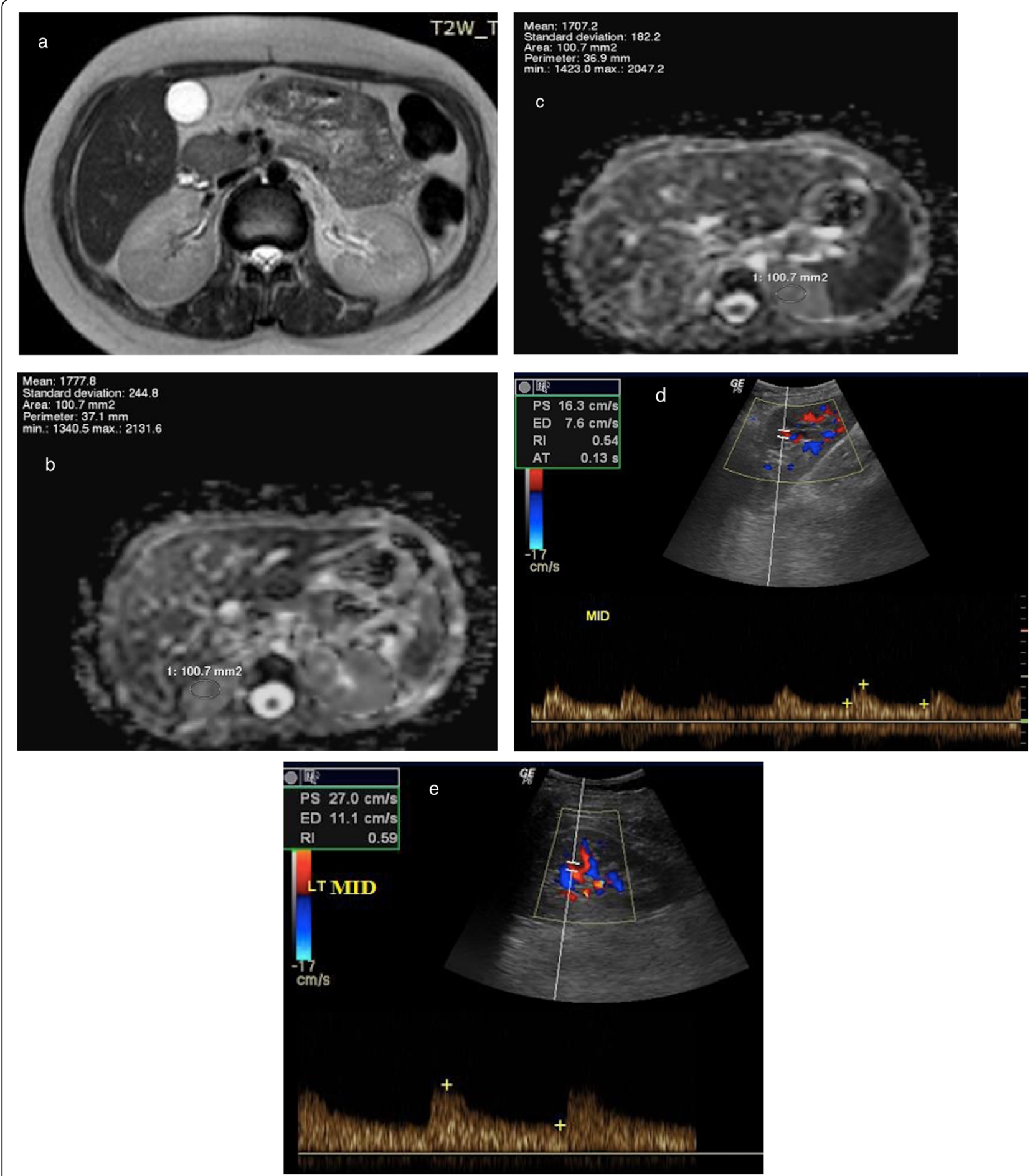

Fig. 1 A 20-month-old male child with CKD stage 2, due to typical HUS. a axial T2 Wls of the abdomen showing mildly increased T2 signal intensity. b, c ADC map and measurement of ADC values: right kidney (b) -1.8 , left kidney (c) $-1.7 \times 10^{-3} \mathrm{~mm}^{2} / \mathrm{s}$. d, e Color Doppler and spectral wave showing segmental Rl of right (d) and left (e) kidney

\section{Results}

This study included 20 patients with CKD and 10 control cases without any known renal disease. A total of 60 kidneys from 30 subjects were included in this study.
The study population was classified into two groups: Group A: CKD due to any renal parenchymal disease (40 kidneys) and group B: Healthy control subjects (20 kidneys). 

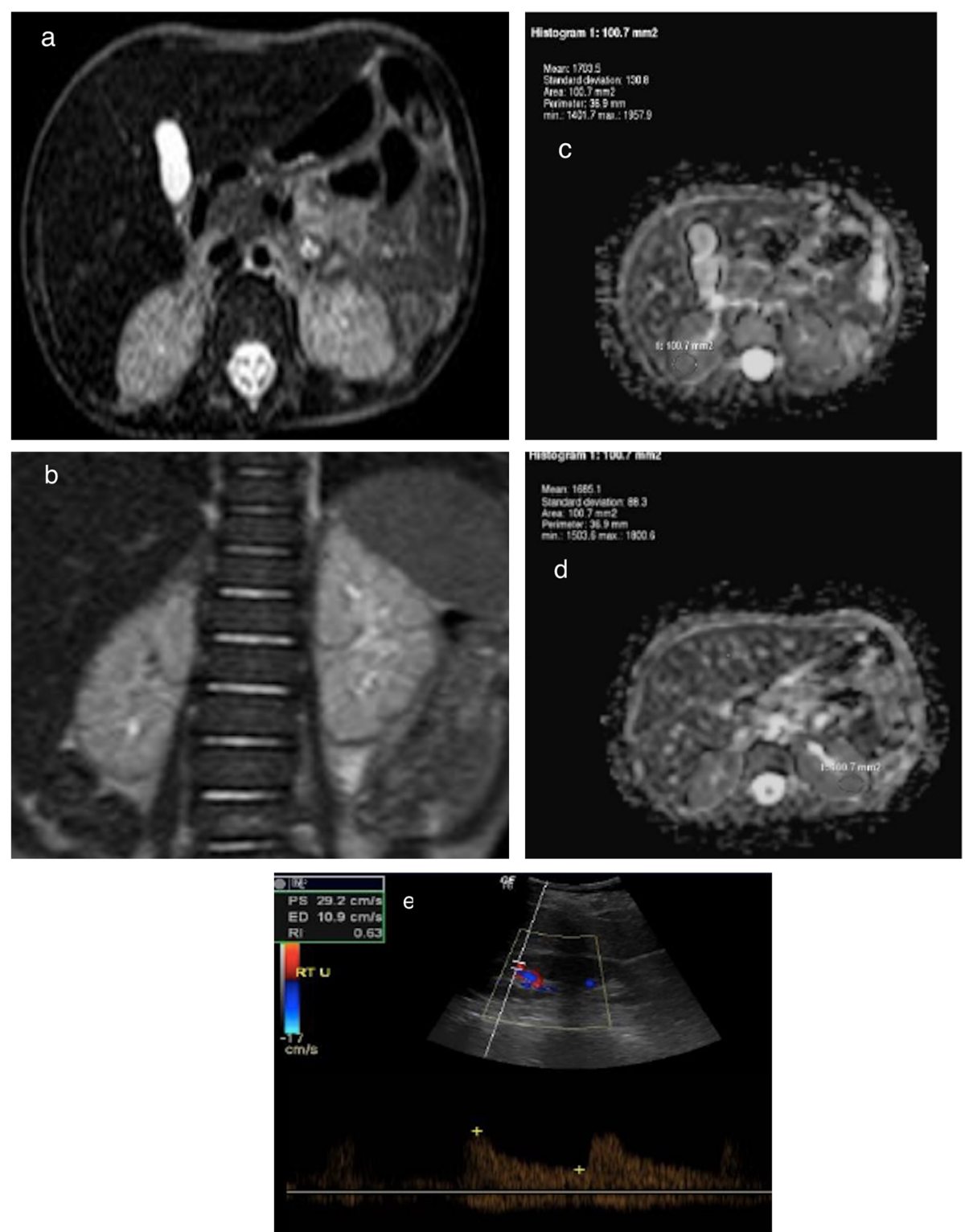

Fig. 2 A 22-month-old male child with CKD stage 5, due to atypical HUS who underwent plasmapheresis 5 times. a axial and b coronal T2 Wls of the abdomen showing increased parenchymal T2 signal of both kidneys with decreased corticomedullary differentiation. $\mathbf{c}$ ADC right kidney $=$ $1.7 \times 10 \mathrm{~mm} / \mathrm{s}$. d ADC left kidney $=1.6 \times 10 \mathrm{~mm} / \mathrm{s}$. e Renal Doppler images of right kidney with $\mathrm{Rl}=0.63$

\section{Clinical findings}

The etiology of CKD is established by the pediatric nephrologist based on clinical and imaging findings as well as pathology reports. Nine patients (45\%) had glomerulonephritis, 5 patients (25\%) had hemolytic uremic syndrome (Fig. 1), 2 patients (10\%) had lupus nephritis, 2 patients (10\%) had nepheronophthisis, and 1 patient $(1.5 \%)$ had infantile nepherosis, whereas the cause of CKD was unknown in one patient (1.5\%).

The stages of CKD were classified according KIDGO guidelines: 6 patients (30\%) were stage 1, 4 patients (20\%) were stage 2,3 patients $(15 \%)$ were stage 3,2

Table 1 Distribution of ADC in the study groups

\begin{tabular}{lllllll}
\hline & CKD patients (group A) & & & Control (group B) \\
\hline ADC & Mean \pm SD & Min & Max & Mean \pm SD & Min & Max \\
& $1.85 \pm 0.24$ & 1.2 & 2.3 & $2.2 \pm 0.12$ & 2.5 \\
\hline
\end{tabular}


Table 2 Correlation between ADC, RI, and stage of CKD in group A

\begin{tabular}{|c|c|c|c|c|c|}
\hline \multicolumn{2}{|c|}{ Children with CKD } & \multirow{2}{*}{$\frac{\text { Stage of CKD }}{1.000}$} & \multirow{2}{*}{$\begin{array}{l}\text { Doppler (RI) } \\
.123\end{array}$} & \multirow{2}{*}{$\frac{\mathrm{eGFR} \mathrm{ml} / \mathrm{min} / 1.73 \mathrm{~m}^{2}}{-.974}$} & \multirow{2}{*}{$\frac{\mathrm{ADC}}{-.655}$} \\
\hline Stage of CKD & Correlation coefficient & & & & \\
\hline & $P$ value & - & .448 & $<0.001$ & $<0.001$ \\
\hline \multirow[t]{2}{*}{ Doppler (RI) } & Correlation coefficient & .123 & 1.000 & -.172 & -.280 \\
\hline & $P$ value & .448 & - & .289 & .080 \\
\hline \multirow[t]{2}{*}{$A D C$} & Correlation coefficient & -.655 & -.280 & .696 & 1.000 \\
\hline & $P$ value & $<0.001$ & .080 & $<0.001$ & - \\
\hline
\end{tabular}

patients (10\%) were stage 4 , and 5 patients (25\%) were stage 5 (Fig. 2).

\section{Imaging findings}

\section{Doppler US findings}

In group $\mathrm{A}, \mathrm{RI}$ ranges from 0.46 to 1 with mean $=0.66 \pm$ 0.11 . The RI in group $B$ ranged from 0.49 to 0.64 , with a mean of $0.57 \pm 0.05$. There was a significant difference of the mean RI value between each group $(P$ value $<0.001)$.

\section{DWI Findings}

The mean ADC value of the kidney in group A was 1.85 $\times 10^{-3} \pm 0.24$, with a minimum of 1.20 and a maximum of 2.30. In group $\mathrm{B}$, the mean $\mathrm{ADC}$ was $2.21 \times 10^{-3} \pm$ 0.12 , with a minimum of 2.0 and a maximum of 2.5 (Table 1). There was a significant difference between the mean ADC values of the patients and control group $(P$ value $<0.00)$.

\section{Correlation of $A D C, R I$, and stages of CKD (Table 2)}

The relationship between the stage of CKD and RI showed negligible positive correlation (' $r$ ' value $=0.123$, $p$ value $=0.448)$. As for the correlation between the stage of CKD and ADC, we found it to be a moderate negative one with $r^{\prime}$ value of -.655 and a significant $p$ value of $<0.001$. Lastly, the correlation between the RI and mean ADC was a weak negative one (' $r$ ' value $=-.280, p$ value $=0.080)$ (Figs. 3 and 4$)$.

\section{Discussion}

Evaluation of renal microstructure is pivotal for diagnosing and monitoring chronic renal disease, being currently performed by an invasive procedure like renal biopsy with its risk of complication and sampling errors [15]. DWI has been proved to be practicable and reliable examination for the evaluation of renal function and parenchymal damage in some renal diseases [16-20].

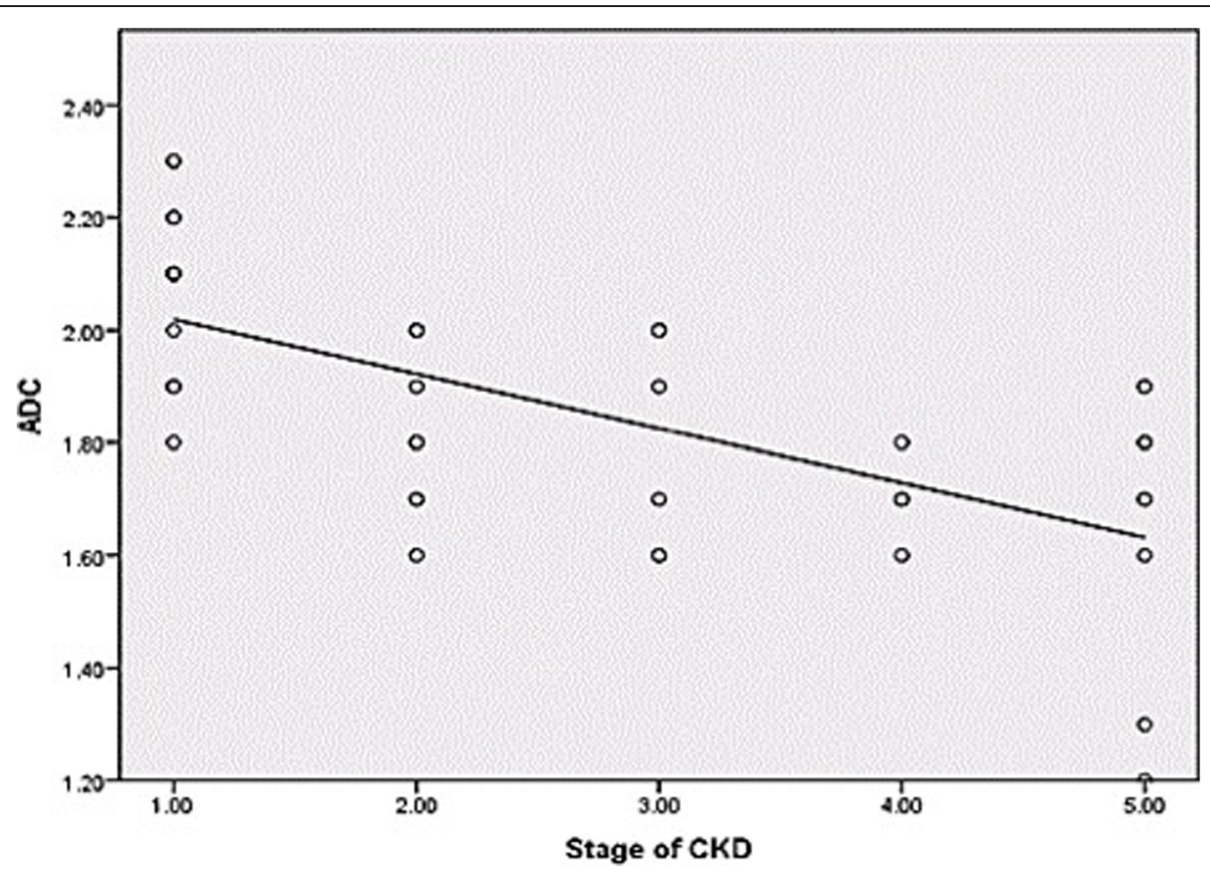

Fig. 3 Scattered plots depicting relation of changes in ADC value and stage of CKD 


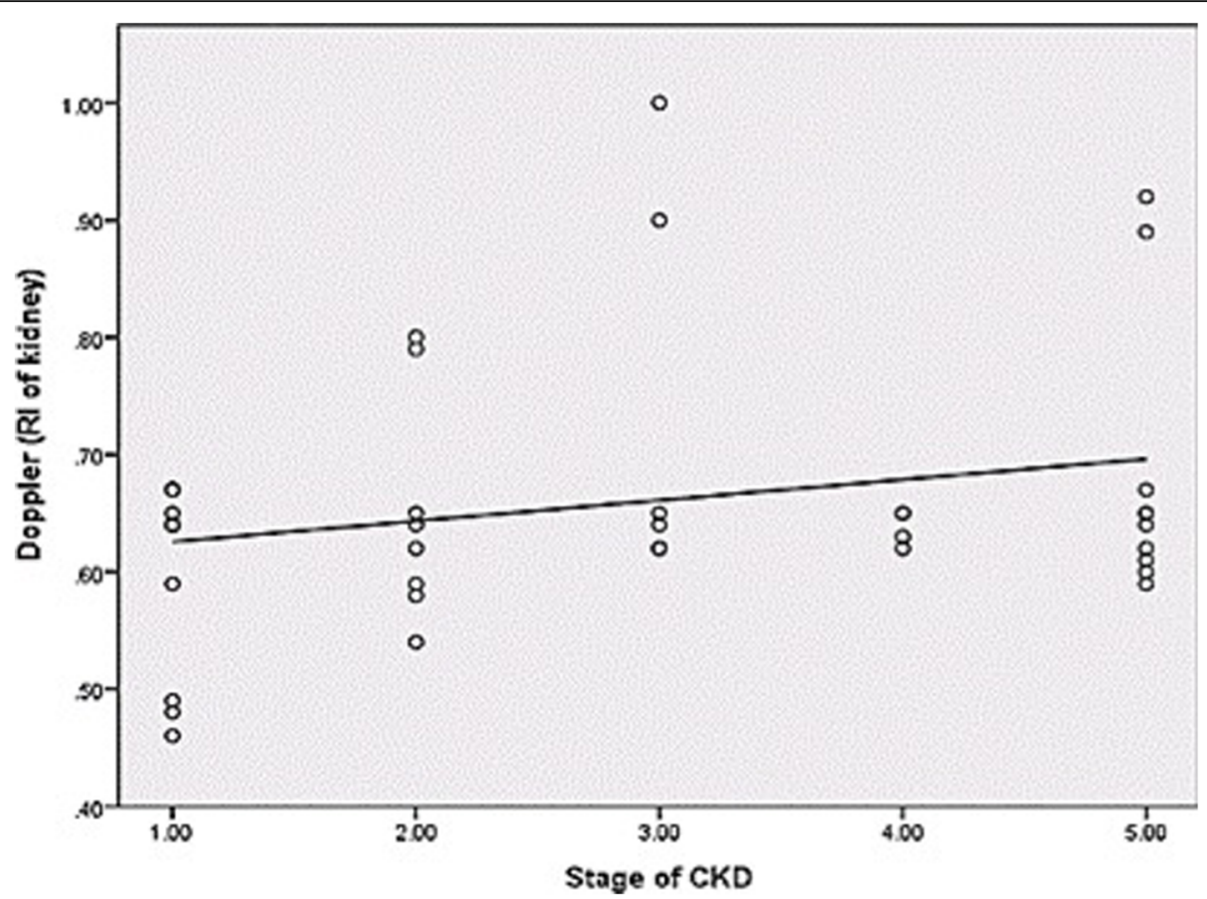

Fig. 4 Scattered plots depicting relation of changes in RI of segmental renal artery and stage of CKD

In this study, we aim to evaluate the role of DWI in the assessment CKD and investigate the correlation between different stage of the CKD and renal parenchymal ADC value changes.

The optimal $b$ value for abdominal DWI has not been determined exactly. In this study, we performed DWI sequence using $b$ values of 0 and $600 \mathrm{~s} / \mathrm{mm}^{2}$, which has been used in several previous studies $[4,5,8,20]$ to yield a sufficient SNR, reduce "T2 shine through" and avoid perfusion effect.

In all cases and control, DW sequence was performed during free breathing. Kocyigita et al. in their study on 46 children with VUR reported reproducible ADC values on DWI without pulse triggering during free breaching, which is a considerable advantage in children [8].

In some of the previously published studies $[19,21]$, the authors had evaluated the ADC of renal cortex and renal medulla in each kidney and had reported a significant difference between ADC value of renal cortex and medulla. Because of lower resolution of images with higher $b$ values, it was difficult to reliably discriminate between cortex and medulla; hence, ROIs were placed on the renal parenchyma. This was supported by the findings of Thoeny et al. [22] and Xu et al. [23], and they have reported that there is no statistical difference between the ADC values of the cortex and medulla.

The mean ADC value of renal parenchyma in our patients with CKD was $\left(1.85 \pm 0.24 \times 10-^{3} \mathrm{~mm}^{2} / \mathrm{s}\right)$ which is significantly lower than that of the control cases $(2.21 \pm$ $0.12 \times 10-^{3} \mathrm{~mm}^{2} / \mathrm{s}$ ) with $p$ value $<0.001$. This is similar to the findings of several previous studies [21, 23, 24]. The low renal parenchyma ADC values in CKD are due to reduced perfusion as well as reduced water diffusion. Different pathological factors including glomerulosclerosis, interstitial fibrosis, and tubular atrophy restrict the free water molecules movement in both the extracellular and intracellular space [4].

The results of this study were in agreement with several authors who have reported that renal ADC value has a potential correlation with renal function, showing lower ADC values with a decrease in GFR [23, 24]. Thus, DWI can be utilized as non-invasive technique to monitor renal function changes [25].

A study by $\mathrm{Xu}$ et al. on a 1.5 -T MR unit with $b$ values of 0 and 500 seconds $/ \mathrm{mm}^{2}$ found that the ADC was significantly lower in impaired kidneys than in normal kidneys, and there was a positive correlation between ADCs and split GFR $(r=0.709)$ [10].

In another study performed on 72 healthy volunteers and 43 patients with CKD (chronic glomerulonephritis) using $b$ value 0 and $500 \mathrm{~s} / \mathrm{mm}^{2}$, they have found that patients with CKD had significantly lower renal ADC $(r=-4.383, p=0.000)$ than age and sexmatched volunteers, and a negative correlation was found between ADC and stages of CKD $(r=-$ 0.492, $p=0.000$ ) [23].

In our study, the mean ADC values of kidneys in patients with CKD were significantly lower than normal at most stages of CKD, except in stage 1 . We found an overlap of $\mathrm{ADC}$ value in stage $1 \mathrm{CKD}$ patients with that 
of the normal control. This was in concordance with the findings of other previous studies [23, 24].

We have found that the mean RI of group A $(0.66 \pm$ .11) was significantly higher than that of group B $(0.57 \pm$ .05) with $p$ value $<0.001$. This was matching with the findings of Hanamura and his colleagues who have reported increased RI with the progression of CKD stage. Additionally, RI was correlated with age, eGFR, and renal histological changes, including glomerulosclerosis, arteriosclerosi,s and tubulointerstitial damage. They concluded that RI in CKD patients was considered a marker for assessment of renal function, histological damage and disease prognosis as well as a possible determinant of indication for steroid [26].

Sugiura and colleagues also stated that high RI (> 0.70) was an independent risk factor for renal function deterioration in CKD, and elevated RI was linked to lower renal survival [27].

In our study, there was strong positive correlation between RI and stage of CKD in group A. This was consistent with the findings of other studies [26, 28] including Parolini et al. who showed that the patients with an initial RI of 0.70 or higher showed a rapid decline of renal function independent of initial eGFR. Initial RI in their study not only correlated significantly with eGFR of the initial groups representing its correlation with CKD stage but also with final eGFR on follow-up [29].

There are few limitations in our study: First, relatively small sample size with limited cases of each cause (e.g. HUS, LN). Second, wide range of pediatric population ranging from age 2 to 18 years, with the ADC not compared with the control group of the respective age group. Lastly, no clear cutoff of ADC values can be used in the differentiation between CKD and normal kidney. Further studies are warranted to evaluate the diagnostic accuracy of DW MRI in a larger pediatric patient cohort compared to healthy volunteers of a wide age range.

\section{Conclusion}

Our study concluded that DWI is a promising imaging tool that can take part in the assessment of the morphological and functional changes in diffuse renal parenchymal disease, hence playing an important role in the early diagnosis and staging of chronic kidney disease.

\section{Abbreviations}

CKD: Chronic kidney disease; eGFR: Estimated glomerular filtration rate; USG: Ultrasonography; CT: computed tomographic; DWI: Diffusion-weighted imaging; RI: Resistivity index (RI)

\section{Acknowledgements}

The authors wish to acknowledge the assistance of MR operators in Cairo University Hospitals.

\section{Authors' contributions}

S.E. contributed in the study conception and design and wrote and edited the manuscript. S.Y. collected, analyzed, and interpreted the patient data. R.G. contributed in referral and follow-up of the cases. W.E. contributed in the study conception and wrote and edited the manuscript. All authors read and approved the final manuscript.

Funding

This study was self-funded.

\section{Availability of data and materials}

The datasets used and/or analyzed during the current study are available from the corresponding author on reasonable request.

Ethics approval and consent to participate

The study was approved by the ethics committee of faculty of medicine, Cairo University. Reference number is not applicable. Informed written consents were obtained from guardian of children before the examination.

\section{Consent for publication}

All guardians of the children included in this research gave written informed consent to publish the data contained within this study.

\section{Competing interests}

The authors declare that they have no competing interests.

\section{Author details}

${ }^{1}$ Diagnostic and Intervention Radiology Department, Cairo University Hospitals, Kasr Al-Ainy, El-Manial, Cairo 11956, Egypt. ²Pediatric Department, Nephrology Unit, Cairo University Hospitals, Kasr Al-Ainy, Cairo, Egypt.

Received: 11 June 2020 Accepted: 3 August 2020

Published online: 27 August 2020

\section{References}

1. Becherucci F, Roperto RM, Materassi M, Romagnani P (2016) Chronic kidney disease in children. Clin Kidney J 9(4):583-591

2. Chandarana H \& Lee VS. Renal functional MRI: are we ready for clinical application? Am J Roentgenol. Jun 1, 2009;192(6):1550-1557.

3. Yalcin-Safak K, Ayyildiz M, Unel SY, Umarusman-Tanju N, Akca A, Baysal T (2015) The relationship of ADC values of renal parenchyma with CKD stage and serum creatinine levels. Eur J Radiol 3:8-11

4. Goyal A, Sharma R, Bhalla AS, Gamanagatti S, Seth A (2012) Diffusion weighted MRI in the assessment of renal dysfunction. Indian J Radiol Imaging 22(3):155-159

5. Cova M, Squillaci E, Stacul F, Manenti G, Gava S, Simonetti G, et al. Diffusionweighted MRI in the evaluation of renal lesions: preliminary results. $\mathrm{Br}$ J Radiol. Oct 1, 2004;77(922):851-857.

6. Lin F, Li Z, Gan Y, Sun L, Fang D, Xiang K et al (2014) Relationship between renal apparent diffusion coefficient values and glomerular filtration rate in infants with congenital hydronephrosis. Biosci Trends 8:274-279

7. Jones RA, Grattan-Smith JD (2003) Age dependence of the renal apparent diffusion coefficient in children. Pediatr Radiol 33:850-854

8. Kocyigita A, Bayrama R, Selcuk Y, Yilmaz I, Karabulut N (2014) Diffusion weighted magnetic resonance imaging of kidneys in children with vesicoureteral reflux. Eur J Radiol 83:e56-e60

9. Bedoya MA, Jeffrey I, Berman JI, Delgado J et al (2019) Relationship of renal apparent diffusion coefficient and functional MR urography in children with pelvicalyceal dilation. Pediatr Radiol 49:1032-1041

10. Levey AS, Coresh J, Bolton K, Culleton B, Harvey KS, Ikizler TA, et al.; National Kidney Foundation. KDOQI Clinical practice guidelines for chronic kidney disease: evaluation, classification and stratification., Am J Kidney Dis. 2002. Vol. 39

11. Harambat J, Van Stralen KJ, Kim JJ, Tizard EJ (2012) Epidemiology of chronic kidney disease in children. Pediatr Nephrol 27(3):363-373

12. Chan YH (2003) Biostatistics 102: Quantitative data - parametric \& nonparametric tests. Singap Med J 44(8):391-6. 113

13. Chan YH (2003) Biostatistics 103: qualitative data-tests of independence. Singap Med J 44(10):498-503

14. Chan YH (2003) Biostatistics 104. Singapore Med J 44(12):614-619 
15. Caroli A, Schneider M, Friedly I, Ljimani A, De Seingneux S, Boor P et al (2018) Diffusion-weighted magnetic resonance imaging to assess diffuse renal pathology: a systematic review and statement paper. Nephrol Dial Transplant 33:ii29-ii40

16. Yildirim E, Kirbas I, Teksam M, Karadeli E, Gullu H, Ozer I (2008) Diffusionweighted MR imaging of kidneys in renal artery stenosis. Eur J Radiol 65: 148-153

17. Bozgeyik Z, Kocakoc E, Sonmezgoz F (2009) Diffusion-weighted MR imaging findings of kidneys in patients with early phase of obstruction. Eur J Radiol 70:138-141

18. Zheng Z, Yan T, Jia J, Li D, Wei L, Shang W et al (2018) Assessment of renal pathological changes in lupus nephritis using diffusion weighted imaging: a multiple correspondence analysis. Kidney Blood Press Res 43(3):847-859

19. Herrmann J, Wenzel U, Galler S, Schoennagel BP, Busch JD, Tozakidou M et al (2017) Diffusion weighted imaging of the kidneys in haemolytic uraemic syndrome. Eur Radiol 27(11):4591-4601

20. Cakmak P, Yagc AB, Dursun B, Herek D, Fenkci SM (2014) Renal diffusionweighted imaging in diabetic nephropathy: correlation with clinical stages of disease. Diagn Interv Radiol 20:374-378

21. Yoshikawa T, Kawamitsu H, Mitchell DG, Ohno Y, Ku Y, Seo Y et al (2006) $A D C$ measurement of abdominal organs and lesions using parallel imaging technique. Am J Roentgenol 187(6):1521-1530

22. Thoeny HC, De Keyzer F, Oyen RH, Peeters RR (2005) Diffusion-weighted MR Imaging of Kidneys in Healthy Volunteers and Patients with Parenchymal Diseases: Initial Experience. Radiology. 235(3):911-917

23. Xu X, Fang W, Ling H, Chai W, Chen K (2010) Diffusion-weighted MR imaging of kidneys in patients with chronic kidney disease: initial study. Eur Radiol 20(4):978-983

24. Xu Y, Wang X, Jiang X (2007) Relationship between the renal apparent diffusion coefficient and glomerular filtration rate: Preliminary experience. J Magn Reson Imaging 26(3):678-681

25. Liu H, Zhou Z, Li X, Li C, Wang R, Zhang U et al (2018) Diffusion weighted imaging for staging chronic kidney disease: a meta-analysis. Bjr J Radiol 91 1091

26. Hanamura K, Tojo A, Kinugasa S, Asaba K, Fujita T (2012) The resistive index is a marker of renal function, pathology, prognosis, and responsiveness to steroid therapy in chronic kidney disease patients. Int J Nephrol 4:139565

27. Sugiura T, Wada A (2009) Resistive index predicts renal prognosis in chronic kidney disease. Nephrol Dial Transplant 24(9):2780-2785

28. Making Y, Ogawa M, Ueda S, Hori J, Ohto M, Wakashin M et al (1992) Intrarenal arterial Doppler sonography in patients with various renal disease: Correlation of resistive index with biopsy findings. Japanese J Nephrol 34(2): 207-212

29. Parolini C, Noce A, Staffolani E, Giarrizzo GF, Costanzi S, Splendiani G (2009) Renal resistive index and long-term outcome in chronic nephropathies. Radiology. 252(3):888-896

\section{Publisher's Note}

Springer Nature remains neutral with regard to jurisdictional claims in published maps and institutional affiliations.

\section{Submit your manuscript to a SpringerOpen ${ }^{\circ}$ journal and benefit from:}

- Convenient online submission

- Rigorous peer review

- Open access: articles freely available online

- High visibility within the field

- Retaining the copyright to your article

Submit your next manuscript at $\boldsymbol{\nabla}$ springeropen.com 